\title{
Sexual risk behavior among HIV-positive persons in Jamaica.
}

\author{
Butho Ncube ${ }^{1}$, Jessica Ansong ${ }^{1}$, Kewanna Daniels ${ }^{1}$, Dianne Campbell-Stennett ${ }^{2}$, Pauline E Jolly ${ }^{1}$
}

1. Department of Epidemiology, University of Alabama at Birmingham, Birmingham, AL, United States

2. Ministry of Health, Westmoreland Health Department, Jamaica

\begin{abstract}
Background: HIV/AIDS remains a global public health challenge, especially in sub-Saharan Africa and the Caribbean. Sexual risk behaviors among HIV-positive persons place their partners at risk for HIV transmission and other sexually transmitted infections. Stopping transmission acts among HIV-positive people is crucial in reversing HIV incidence.

Objective: This study aimed to assess the prevalence and predictors of sexual risk behaviors among HIV-positive individuals in clinical care in Northwestern Jamaica.

Methods: A cross-sectional survey of 118 (33 males and 85 females) HIV-positive individuals was used to assess demographic and health characteristics, HIV/AIDS knowledge, attitudes, and beliefs and sexual risk behaviors.

Results: About $12 \%$ of the study population stated that they had unprotected anal or vaginal sex without disclosing their HIV status. Participants who agreed that condoms reduce the risk of HIV transmission were 13.1 times more likely to use condoms during their last sexual encounters(95\% CI: 2.1-79.0) than those who disagreed. About $75 \%$ of participants reported using a condom every time they had sexual intercourse in the past year, while $25 \%$ used condoms irregularly. Participants who had unprotected anal or vaginal sex without disclosing their status were less likely to have used condoms during the last sexual encounter (OR=0.1; 95\% CI: 0.02-0.5).

Conclusion: The prevalence of unsafe sex remains high among sexually active people living with HIV/AIDS in Jamaica. Study participants who engaged in unprotected sex without disclosing their HIV-positive status potentially place their partners at risk for HIV transmission and other sexually transmitted infections. The study findings highlight the need to promote safe sexual behaviors and a positive social environment for people living with HIV/AIDS in Jamaica.
\end{abstract}

Keywords: HIV-seropositivity, sexual behavior, Jamaica, anti-retroviral therapy, condom use.

DOI: https://dx.doi.org/10.4314/ahs.v17i1.6

Cite as: Ncube B, Ansong J, Daniels K, Campbell-Stennett D, Jolly PE. Sexual risk behavior among HIV-positive persons in Jamaica. Afri Health Sci. 32-38. bttps:/ / dx.doi.org/10.4314/abs.v17i1.6

\section{Introduction}

Studies show that one third of HIV-positive people continue to practice unprotected sexual practices after testing positive. ${ }^{1,2}$ This potentially places partners of HIV positive people at risk for HIV transmission and other sexually transmitted infections. Moreover, HIV-positive people risk re-infection with new HIV strains or other sexually transmitted infections. ${ }^{1}$ Stopping transmission acts

\footnotetext{
Corresponding author:

Pauline E Jolly,

Department of Epidemiology,

School of Public Health,

University of Alabama at Birmingham,

Ryals Building, Room 217

Birmingham, Alabama 35294-0022

Tel: 2059341823

Fax: 2059753329

Email: jollyp@uab.edu
}

among HIV-positive people is crucial in reversing HIV incidence. According to UNAIDS/WHO, the AIDS epidemic claimed an estimated 11, 000 lives in the Caribbean in 2013, making it one of leading causes of death among adults. ${ }^{3}$ In Jamaica, the epidemic has spread from highrisk groups (homosexual men and sex workers) to the general populations. ${ }^{4}$ The HIV prevalence rate in Jamaica is $1.8 \%$, however, prevalence rates higher than $20 \%$ have been reported among the high-risk groups (homosexual men and sex workers). $5,6,7,8$

The HIV/AIDS epidemic has spread to all 14 parishes in Jamaica, with a cumulative AIDS case rate of 468.2 per 100, 000 population. According to the Jamaican Ministry of Health, AIDS's case rates are highest in the parish of St. James (1050.0 per 100, 000 population) which includes Montego Bay, the tourism capital of Jamaica. ${ }^{8}$ In fact, over two million visitors come to Jamaica each year and some tourists engage in sexual activities with Jamaicans. ${ }^{7}$ Few studies have been conducted with respect to the in- 
cidence and behavioral factors related to HIV transmission among HIV-positive individuals in Jamaica. Studies in Jamaica have reported social, cultural, economic and political factors as predictors of sexual risk behaviors. ${ }^{4-9}$ Behavioral factors that have been shown to drive the HIV epidemic in Jamaica include multiple sexual partnerships, early sexual debut, high levels of transactional sex, commercial sex and inadequate condom use., ${ }^{4,6,7}$ The prevalence of unsafe sex among sexually active PLWHA has been documented to be high in other developing nations. ${ }^{10-12}$ A South African study found that half of PLWHA had vaginal or anal sex after testing positive for HIV, and $30 \%$ of these participants reported unprotected vaginal or anal sex. ${ }^{10}$

According to the Jamaican National HIV/STI Control Programme, approximately $80 \%$ of people living with HIV reported having multiple sex-partners. ${ }^{8}$ Furthermore, there is a strong association between STIs and HIV infection in Jamaica. For instance, more than a third $(39 \%)$ of persons living with HIV/AIDS in Jamaica had history of a sexually transmitted infection. ${ }^{4}$ Therefore, people living with HIV need to consistently practice safer sexual behaviors in order to reduce the spread of HIV and further development of drug-resistant HIV strains. ${ }^{13}$ A wide range of factors influence unprotected sex among HIV-infected people. For instance, studies show that condom use fatigue, HIV transmission myths, lack of HIV knowledge, and a desire to have children may lead to PLWHA ignoring the risks of unprotected sex..$^{1,14,15}$ Conversely, self-efficacy regarding safe sex, and motivation to protect partners as well as themselves against re-infection with new HIV strain or other STIs may lead to safe sex practices among HIV-positive individuals. ${ }^{15}$

Expanded access to anti-retroviral drugs (ARV) has enabled people with HIV to live longer and healthier lives. Consequently, the increasing number of sexual transmissions of HIV may result from those who know their HIV-seropositive status but engage in sexual risk behavior. ${ }^{16}$ Persons living with HIV, including those on ARV therapy, must be counselled to practice safe sex, which is known as positive prevention. Positive prevention is considered a key strategy for preventing new infections as it helps to break the chain of HIV transmission. ${ }^{7,17}$ "Healthy relationships" is one of many HIV prevention behavioral interventions that has proven effective in reducing HIV risk associated with unprotected sex. ${ }^{18,19}$
"Healthy relationships" is a small group HIV intervention program based on the social cognitive theory. ${ }^{19}$ The program assists people living with HIV to practice safe sex, manage HIV status disclosure and stigma. ${ }^{18,19}$ Healthy relationship programs have been implemented in almost all states in the United States and many other nations. ${ }^{19}$ However, some intervention activities of healthy relationship programs may not apply to local contexts and cultures in many developing countries, compromising the success of the intervention. ${ }^{18}$

Clearly, there is a need to assess factors associated with sexual risk behavior among PLWHA and design culturally relevant interventions for HIV-positive individuals. Changes in the behavior of HIV-positive people can significantly reduce the spread of the epidemic, thus HIV-positive targeted interventions will be potentially more cost effective.

\section{Objectives}

The objectives of this study were to:

1. Determine socio-economic, attitudes and psychological factors that influence HIV-positive people to engage in risky transmission behavior;

2. Determine the prevalence of high risk sexual behavior among HIV-positive people in order to develop risk reduction interventions;

3. Make recommendations for strategies that can be implemented to stop transmission acts among HIV-seropositive people in order to reduce HIV incidence.

\section{Methods}

Design: convenience sample.

Location and participation: The study was conducted in the parishes of Westmoreland, St. James and Hanover in Western Jamaica PLWHA were the sampling unit of this study. Potential participants were defined as adults 19 years and older who attended HIV treatment sites and agreed to be interviewed. To eliminate the bias created by leaving out PLWHA that did not attend HIV treatment sites, contact investigators called or mailed potential participants to ask for their permission to visit and conduct the study with them. The purpose of the study was explained to potential participants and they were asked to participate. Those who expressed a willingness to participate were asked to read and sign an informed consent form. If the potential participants were unable to read, the consent form was read to them and they were 
asked to give a thumbprint signifying their consent. Participation in this study was voluntary and no incentives were provided. The Institutional Review Board (IRB) of the University of Alabama at Birmingham, the Advisory Panel of Ethics and Medico-Legal Affairs in the Ministry of Health, and the Western Regional Health Authority approved the study protocol prior to its implementation. Sample Size: The study had a total 118 participants, 85 were females and 33 were males aged 19 to 72 years.

\section{Data collection}

Participant views were assessed utilizing an interviewer-administered questionnaire. The questionnaire covered the following topics: demographics; health information (including current health status, year of HIV diagnosis, whether participants had experienced any symptoms of HIV infection or had been diagnosed with an AIDS related condition, and most recent $\mathrm{CD}^{+}$cell count); sexual risk behaviors (number of sexual partners in the previous 3 months, partners' HIV status, condom use, treatment of sexually transmitted infections after testing HIV positive); HIV treatment beliefs; HIV transmission risk perceptions; and psychological state including current attitudes towards life and disclosure of HIV-positive status to family and sexual partners.

\section{Statistical analysis}

Differences in socio-demographic and health characteristics, HIV knowledge, attitude and sexual practices were assessed by gender using chi-square and Fisher's exact tests. Variables that were statistically significant in bivariate analysis and those known to be associated with condom use based on the literature were entered into a multivariate model and Odds Ratios and 95\% confidence intervals calculated. Data analysis was performed using SPSS version 19.0 and SAS software version 9.2.

\section{Results}

\section{Sociodemographic and health characteristics.}

Our study had a total of 118 participants, 72\% (85) were females between the ages of 20 to 66 years and $28 \%$ (33) were males aged 19 to 72 years. The majority (73\%) of the participants completed secondary school. A higher proportion of men than women were employed $(72 \%$ vs. $40 \%)(p=0.001)$. The majority $(61 \%)$ of the participants were single, $33 \%$ were married. A higher proportion of men than women were on ARV treatment (62\% vs. $43 \%)$, had experienced HIV symptoms (38\% vs. $26 \%$ ), and had an HIV-related hospitalization (21\% vs. $14 \%$ ) (Table 1$)$. 
Table 1: Socio-demographic and health characteristics of participants by gender

\begin{tabular}{|c|c|c|c|}
\hline Variables & $\begin{array}{l}\text { Male } \\
{[\mathrm{N}=33(28 \%)]}\end{array}$ & $\begin{array}{l}\text { Female } \\
{[\mathrm{N}=85(72 \%)]}\end{array}$ & p-value \\
\hline \multicolumn{4}{|l|}{ Age } \\
\hline$<35$ & $13(39.4)$ & $48(56.5)$ & 0.08 \\
\hline $35-44$ & $14(42.4)$ & $19(23.8)$ & \\
\hline$\geq 45$ & $6(18.2)$ & $18(17.3)$ & \\
\hline \multicolumn{4}{|l|}{ Education } \\
\hline Primary education or less & $8(24.2)$ & $23(27.4)$ & 0.72 \\
\hline Secondary education or higher & $25(75.8)$ & $61(72.6)$ & \\
\hline \multicolumn{4}{|l|}{ Employment } \\
\hline No & $9(27.3)$ & $51(60.0)$ & 0.001 \\
\hline Yes & $24(72.7)$ & $34(40.0)$ & \\
\hline \multicolumn{4}{|l|}{ Marital status } \\
\hline Single & $20(60.6)$ & $51(60.0)$ & 0.57 \\
\hline Married or living in union & $12(36.4)$ & $27(31.8)$ & \\
\hline Divorced or widowed & $1(3.0)$ & $7(8.2)$ & \\
\hline \multicolumn{4}{|l|}{ ARV } \\
\hline No & $13(39.4)$ & $46(56.1)$ & 0.1 \\
\hline Yes & $20(60.6)$ & $36(43.9)$ & \\
\hline \multicolumn{4}{|l|}{ Have had a STI } \\
\hline No & $31(96.9)$ & $80(96.4)$ & 0.89 \\
\hline Yes & $1(3.1)$ & $3(3.6)$ & \\
\hline \multicolumn{4}{|c|}{ Have experienced HIV symptoms } \\
\hline No & $22(66.7)$ & $62(73.8)$ & 0.44 \\
\hline Yes & $11(33.3)$ & $22(26.2)$ & \\
\hline \multicolumn{4}{|l|}{ HIV-related hospitalization } \\
\hline No & $26(78.8)$ & $72(85.7)$ & 0.36 \\
\hline Yes & $7(21.2)$ & $12(14.3)$ & \\
\hline
\end{tabular}

Bold- Statistically significant; Sum of $\mathrm{N}$ for some variables may not equal total $\mathrm{N}$ due to missing responses

Knowledge, attitude and practice of participants regarding HIV sexual risk behavior

The majority of males $(78 \%)$ and females $(74 \%)$ stated that taking ARV treatment made HIV/AIDS less frightening and controllable. More than half (64\%) of the study population stated that having one sex partner will reduce the risk of contracting HIV $(\mathrm{p}=0.005)$. About $88 \%$ of the participants stated that using a condom reduced the chance of contracting HIV. Almost half (47\%) of the participants reported that they had a regular partner; $38 \%$ of the partners were HIV-positive, $24 \%$ were HIV-negative and $38 \%$ did not know their partner's HIV status. About $75 \%$ (24 females and 17 males) of the participants stated that they used condoms every time they had sexual intercourse in the past 12 months compared to $25 \%$ who used condoms irregularly. A fair percentage $(11 \%)$ of the participants reported that they did not use condoms during their last sexual intercourse with their partners. About $12 \%$ of the study population stated that they had unprotected anal or vaginal sex without disclosing their HIV status. Of these persons, 34\% reported that their partners were HIV-negative and 59\% reported that their partners did not know their HIV status.

About $35 \%$ of the participants stated that they had been discriminated or rejected because of their HIV status. A higher proportion of men more than women reported disclosing their HIV status to friends ( $50 \%$ vs. $22 \%$ ) (p $=0.004)$. More than half $(55 \%)$ of participants reported feeling more depressed, however a higher proportion of women (63\%) reported feeling more depressed than males $(40 \%)(p=0.029)($ Table 2$)$. 
Table 2: Knowledge, attitude and practice of participants regarding HIV sexual risk behavior by gender

\begin{tabular}{|c|c|c|c|}
\hline Variables & $\begin{array}{l}\text { Male } \\
{[\mathrm{N}=33(28 \%)]}\end{array}$ & $\begin{array}{l}\text { Female } \\
{[N=85(72 \%)]}\end{array}$ & p-value \\
\hline \multicolumn{4}{|c|}{$\begin{array}{l}\text { Do you think that antiretroviral treatment makes } \\
\text { HIV/AIDS less frightening and } \\
\text { controllable }\end{array}$} \\
\hline True & $18(78.3)$ & $32(74.4)$ & 0.65 \\
\hline False & $2(8.7)$ & $7(16.3)$ & \\
\hline Don't know & $3(13.0)$ & $4(9.3)$ & \\
\hline \multicolumn{4}{|c|}{$\begin{array}{l}\text { Do you think that antiretroviral treatment reduces the } \\
\text { risk of transmitting HIV }\end{array}$} \\
\hline True & $6(27.3)$ & $10(23.3)$ & 0.52 \\
\hline False & $10(45.5)$ & $23(53.5)$ & \\
\hline Don't know & $5(22.7)$ & $10(23.3)$ & \\
\hline \multicolumn{4}{|c|}{$\begin{array}{l}\text { Having one sex partner will reduce your chances of } \\
\text { contracting HIV }\end{array}$} \\
\hline True & $27(81.8)$ & $48(57.1)$ & 0.05 \\
\hline False & $3(9.1)$ & $33(39.3)$ & \\
\hline Don't know & $3(9.1)$ & $3(3.6)$ & \\
\hline \multicolumn{4}{|c|}{$\begin{array}{l}\text { Using a condom every time you have sex reduces your } \\
\text { chances of contracting HIV }\end{array}$} \\
\hline True & $28(87.5)$ & $76(75.3)$ & 0.661 \\
\hline False & $4(12.5)$ & $7(8.0)$ & \\
\hline Don't know & $0(0.0)$ & $1(1.2)$ & \\
\hline \multicolumn{4}{|c|}{ Friends aware of your HIV status } \\
\hline No & $16(50.0)$ & $65(77.4)$ & 0.004 \\
\hline Yes & $16(50.0)$ & $19(22.6)$ & \\
\hline \multicolumn{4}{|c|}{ Depressed in the last month } \\
\hline No & $19(59.4)$ & $31(36.9)$ & $\mathbf{0 . 0 2}$ \\
\hline Yes & $13(40.6)$ & $53(63.1)$ & \\
\hline
\end{tabular}

Bold- Statistically significant; Sum of $\mathrm{N}$ for some variables may not equal total $\mathrm{N}$ due to missing responses

Factors associated with condom use during the last sexual intercourse

Participants who agreed that condoms reduce the risk of HIV transmission were 13.1 times more likely to use condoms during their last sexual encounter (95\% CI: 2.1 79.0) than those who disagreed. Participants who had unprotected anal or vaginal sex without disclosing their status were $90 \%$ less likely to have used condoms during the last sexual encounter (OR=0.1; 95\% CI: 0.02-0.5).

\section{Discussion}

The findings from this study indicate that sexual risk behaviors exist among HIV-positive persons in Jamaica. A fair percentage of participants $(12 \%)$ reported unprotected anal or vaginal sex without disclosing their HIV status to their sexual partners. These participants were less likely to have used condoms during their last sexual intercourse, thereby, increasing the risk of HIV transmission and placing themselves at risk for infection with STIs or other HIV strains.

Our study finding is in accordance with other studies that have found association between HIV status non-disclosure and sexual risk behaviors. ${ }^{14,20,21}$ For instance, studies in Ghana and South Africa found that HIV status disclosure was associated with less unprotected sex. ${ }^{11,14,21}$ Therefore, it is imperative to understand and encourage factors that promote HIV status disclosure in order to reinforce preventative altruism among HIV-positive persons in Jamaica. On the other hand, HIV status non-disclosure has been linked to stigma. ${ }^{22}$ In fact, about $35 \%$ of our study participants stated that they had experienced discrimination or rejection because of their HIV status. Clearly stigma against PLHWA still exists in Jamaica. Studies in Jamaica have shown that most Jamaicans are not sympathetic to HIV-positive individuals, especially homosexu- 
al men or sex workers. ${ }^{2,23}$ Therefore, it is important to improve empathy toward HIV-positive persons living in Jamaica in order to increase HIV status disclosure and promote protective sexual behaviors.

Consistent condom use among HIV-positive individuals is vital in reducing further transmission of HIV. Our study found that $75 \%$ of participants reported using a condom every time they had sexual intercourse in the past year. This finding is consistent with similar studies conducted in Cameroon, Ghana and South Africa. 11,14,21,24 Although many HIV-positive individuals use condoms every time they have sexual intercourse, a considerable number of HIV-positive persons continue to engage in unprotected sexual behaviors that place others at risk for infection and place themselves at risk for co-infection with pathogens that can complicate HIV disease progression. ${ }^{14,16}$ Thus, it is crucial to develop behavioral interventions that will increase safe sex practices among HIV-positive individuals.

Social stigma has been associated with poor adherence to antiretroviral medication and depression..$^{25,26}$ In accordance with similar studies in Jamaica and sub-Saharan Africa, ${ }^{14,23,27}$ we found high rates (55\%) of self-reported depression among HIV-positive persons. Moreover, a higher proportion of women $(63 \%)$ reported feeling depressed more than males $(40 \%)$. This might be due to high caregiver burden, lack of income or access to ARV medication. Unfortunately, depression is linked to poorer clinical outcomes and quality of life in individuals living with HIV/AIDS. ${ }^{23,26}$ Therefore, HIV-positive persons in Jamaica should be screened for depression and counseled when necessary.

\section{Limitations}

Our study had several limitations including convenience sampling and missing data for some variables. All participants were recruited from health care facilities in Jamaica. Thus, these findings may not be generalized to PLWHA in Western Jamaica or other regions of Jamaica. There is also a possibility of recall and social desirability bias. However, our study findings have brought new insights on knowledge, attitudes, practices and sexual risk behaviors among HIV-positive persons in Jamaica.

\section{Conclusion}

In conclusion, our study found that non-disclosure was associated with unprotected sex, while knowing that condoms reduce HIV risk was associated with more condom use. Additionally, the prevalence of unsafe sex remains high among sexually active PLWHA in Jamaica. These findings indicate the need to promote a positive social environment for PLWHA in Jamaica. HIV-positive persons can be encouraged to promote safe sexual behaviors in order to reduce the spread of HIV. Further research on the relationship between depression, stigma, non-disclosure and sexual risk behaviors is needed.

\section{Competing interests}

The authors declare that they have no competing interests.

\section{Acknowledgements}

This study was supported by Minority Health International Research Training (MHIRT) grant T37-MD001448 from the National Institute on Minority Health and Health Disparities, National Institutes of Health, USA, and the Ministry of Health, Jamaica. Its contents are solely the responsibility of the authors and do not necessarily represent the official views of the National Institutes of Health. The authors thank officials at the Westmoreland Health Department, Jamaica, and their research assistant for their help. The authors are grateful to the men and women who participated in the study.

\section{References}

1. Kalichman S, Pellowski J, Turner C. Prevalence of sexually transmitted co-infections in people living with HIV / AIDS: systematic review with implications for using HIV treatments for prevention. Sexually transmitted infections. 2011;87(3):183--190.

2. Norman L, Jimenes J. Sexual stigma and sympathy: Attitudes toward persons living with HIV in Jamaica. Culture, Health \& Sexuality. 2006;8(5):423 pubmed -433.

3. UNAIDS. Global Statistics. Available at: http://www. unaids.org/en/resources/campaigns/2014/2014gapreport/factsheet

4. Olukoga I. Epidemiologic trends of HIV/AIDS in Jamaica. Revista Panamericana de Salud Publica. 2004;15(5):358$-363$.

5. De La Haye W, Pinnock S, Panton M. Profile and pattern of substance abuse in clients diagnosed with HIV in a substance abuse treatment unit general hospital in Jamaica. West Indian Medical Journal. 2004;53:204-211.

6. Duncan J, Gebre Y, Grant Y, Wedderburn M, Byfield 
L, Bourne D et al. HIV prevalance and related behaviors among sex workers in Jamaica. Sexually Transmitted Diseases. 2010;37(5):306-310.

7. Figueroa J, Duncan J, Byfield L, Harvey K, Gebre Y, Hylton-Kong $\mathrm{T}$ et al. A comprehensive response to the HIV/AIDS epidemic in Jamaica: a review of the past 20 years. West Indian Medical Journal. 2008;57(6):562-576.

8. Jamaica Ministry of Health. Jamaica AIDS Report 2010. Kingston:MOH. 2010.

9. White R, Carr R. Homosexuality and HIV/AIDS stigma in Jamaica. Culture, Health \& Sexuality. 2005;7(4):347 pubmed -359 .

10. Bateganya M, Colfax G, Shafer L, Kityo C, Mugyenyi P, Serwadda D et al. Antiretroviral therapy and sexual behavior: A comparative study between antiretroviral-naïve and -experienced patients at an urban HIV/AIDS care and research center in Kampala, Uganda. AIDS Patient Care and STD's. 2005;19(11):760-768.

11. Kien M, Christie S, Cornman D, Fisher W, Shuper P, Pillay $S$ et al. Sexual risk behavior among HIV-positive individuals in clinical care in urban kwazulu-Natal, South Africa. AIDS. 2006;20:1781-1784 pubmed .

12. Sarna A, Luchters S, Geibel S, Kaai S, Munyao P, Shikely $\mathrm{K}$ et al. Sexual risk behavior and HARRT: a comparative study of HIV-infected persons on HAART and on preventive therapy in Kenya. International Journal of STD \& AIDS. 2008;19:85-89.

13. Allen C, Mbonye M, Seeley J, Birungi J, Wolff B, Coutinho, $\mathrm{A}$ et al. ABC for people with HIV: responses to sexual behavior recommendations among people receiving antiretroviral therapy in Jinja, Uganda. Culture, Health \& Sexuality. 2011;13(5):529 pubmed -543.

14. Ncube N, Akunna J, Babatunde F, Nyarko A, Yatich N, Ellis W et al. Sexual risk behaviour among HIV-positive persons in Kumasi, Ghana. Ghana Medical Journal. 2012;46(1).

15. Sarna A, Luchters S, Pickett M, Chersich M, Okal J, Geibel S et al. Sexual behavior of HIV-positive adults not accessing HIV treatment in Mombasa, Kenya: Defining their prevention needs. AIDS Research \& Therapy. 2012;9. 16. Crepaz N, Marks G. Towards an understanding of sexual risk behavior in people living with HIV: a review of social, psychological, and medical findings. AIDS. 2002;16(2):135-149.

17. Wang N, Sun X, Yin L, Liu H, Ruan Y, Shao Y et al. Meta-Analysis of Interventions for Reducing Number of Sexual Partners and Drug and Alcohol Abuse among People Living with HIV/AIDS. Journal of AIDS \& Clinical Research. 2013.

18. Cloete A, Strebel A, Simbayi L, van Wyk B, Henda N, Nqeketo A. Challenges faced by people living with HIV/ AIDS in Cape Town, South Africa: Issues for grouprisk reduction interventions. AIDS Research and Treatment. 2010.

19. Kalichman S, Cherry C, White D, Pope H, Cain D, Kalichman M. Altering key characteristics of a disseminated effective behavioral intervention for HIV positive adults: the "healthy relationships" experience. The journal of primary prevention. 2007;28(2):145-153.

20. Deribe K, Woldemichael K, Wondafrash M, Haile A, Amberbir A. High-risk behaviours and associated factors among HIV-positive individuals in clinical care in southwest Ethiopia. Tropical Doctor. 2008;38(4):237 pubmed $-239$.

21. Simbayi L, Kalichman S, Strebel A, Cloete A, Henda N, Mqeketo A. Disclosure of HIV status to sex partners and sexual risk behaviours among HIV-positive men and women, Cape Town, South Africa. Sexually Transmitted Infections. 2007;83(1):29-34.

22. Mayfield Arnold E, Rice E, Flannery D, RotheramBorus M. HIV disclosure among adults living with HIV. AIDS Care. 2008;20(1):80 pubmed -92.

23. Clarke T, Gibson R, Barrow G, Abel W, Barton E. Depression among persons attending a HIV/AIDS outpatient clinic in Kingston, Jamaica. West Indian Medical Journal. 2010;59(4):369--373.

24. Dia A, Fabienne M, Renée-Cécile B, Sylvie B, Anne-Déborah B, Camelia P et al. Prevalence of unsafe sex with one's steady partner either HIV-negative or of unknown HIV status and associated determinants in Cameroon (EVAL ANRS12-116 survey). Sexually Transmitted Infections. 2010;86(2):148-154.

25. Leserman J. Role of depression, stress, and trauma in HIV disease progression. Psychosomatic Medicine. 2008;70(5):539-545.

26. Schuster R, Bornovalova M, Hunt E. The influence of depression on the progression of HIV: direct and indirect effects. Behavior modification. 2011;36(2):123-145 27. Berhe H, Bayray A. Prevalence of depression and associated factors among people living with HIV/AIDS in Tigray, North Ethiopia: Across sectional hospital based study. International Journal. 2013;4. 